\title{
ERK5 signalling in prostate cancer promotes an invasive phenotype
}

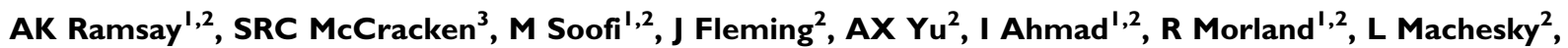 C Nixon ${ }^{2}$, DR Edwards ${ }^{4}$, RK Nuttall ${ }^{4}, M_{\text {Seywright }}{ }^{5}$, R Marquez $^{6}$, E Keller $^{7}$ and HY Leung ${ }^{*, 1,2}$}

'Institute for Cancer Sciences, College of Medical, Veterinary and Life Sciences, University of Glasgow, Glasgow G6I IBD, UK; ${ }^{2}$ Urology Research, The Beatson Institute for Cancer Research, Glasgow Centre for Cancer Research, Garscube Estate, Switchback Road, Bearsden, Glasgow G6I IBD, UK; ${ }^{3}$ Urology Research Group, Northern Institute for Cancer Research, Medical School, University of Newcastle Upon Tyne, Framlington Place, Newcastle Upon Tyne NE2 4HH, UK; ${ }^{4}$ School of Biological Sciences, University of East Anglia, Norwich NR4 7TJ, UK; ${ }^{5}$ Department of Pathology, Western Infirmary, NHS Greater Glasgow and Clyde, Glasgow GII 6NT, UK; ${ }^{6}$ WestCHEM, Department of Chemistry, University of Glasgow, Glasgow G12 8QQ, UK; ${ }^{7}$ Departments of Urology and Pathology, Michigan University, 1500 East Medical Center Drive, 5304 CCGC, Ann Arbor, MI 48109, USA

BACKGROUND: Aberrant mitogen/extracellular signal-regulated kinase 5 (MEK5)-extracellular signal-regulated protein kinase 5 (ERK5)mediated signalling has been implicated in a number of tumour types including prostate cancer (PCa). The molecular basis of ERK5driven carcinogenesis and its clinical relevance remain to be fully characterised.

METHODS: Modulation of ERK5 expression or function in human PCa PC3 and PC3-ERK5 (stably transfected with ERK5) cells was performed using siRNA-mediated knockdown or the MEK inhibitor PDI 8435 respectively. In vitro significance of ERK5 signalling was assessed by assays for proliferation, motility, invasion and invadopodia. Expression of matrix metalloproteinases/tissue inhibitors of metalloproteases was determined by Q-RT-PCR. Extracellular signal-regulated protein kinase 5 expression in primary and metastatic PCa was examined using immunohistochemistry.

RESULTS: Reduction of ERK5 expression or signalling significantly inhibited the motility and invasive capability of PC3 cells. Extracellular signal-regulated protein kinase 5-mediated signalling significantly promoted formation of in vivo metastasis in an orthotopic PCa model $(P<0.05)$. Invadopodia formation was also enhanced by forced ERK5 expression in PC3 cells. Furthermore, in metastatic PCa, nuclear ERK5 immunoreactivity was significantly upregulated when compared with benign prostatic hyperplasia and primary $P C a(P=0.013$ and $P<0.0001$, respectively).

CONCLUSION: Our in vitro, in vivo and clinical data support an important role for the MEK5-ERK5 signalling pathway in invasive PCa, which represents a potential target for therapy in primary and metastatic PCa.

British Journal of Cancer (201 I) I 04, 664-672. doi:I0.1038/sj.bjc.6606062 www.bjcancer.com

Published online 25 January 2011

(c) 201 I Cancer Research UK

Keywords: prostate cancer; signalling; invasive phenotype

Prostate cancer (PCa) is a significant contributor to morbidity and mortality in the Western World. In 2009, there were an estimated 192280 new cases of PCa diagnosed with an estimated $27360 \mathrm{PCa}$ deaths in the United States (Altekruse et al, 2010). Over the last three decades, its incidence has trebled, mostly attributed to improved detection through widespread prostate-specific antigen (PSA) testing. In addition, as life expectancy improves, the rise in incidence is anticipated to continue, worsening its health burden. Currently, treatment selection depends on the prognostic factors of Gleason grade, TNM stage and serum PSA levels, as well as the patient's age, health and own preference. Patients deemed at low risk are recommended active surveillance, but most organconfined disease is treated with curative intent, with surgery or radiation. For locally advanced and metastatic PCa, androgen ablation therapy remains one of the commonest treatment,

*Correspondence: Professor HY Leung;

E-mail: h.leung@beatson.gla.ac.uk

Received 2 September 2010; revised 19 November 2010; accepted 29 November 20 I0; published online 25 January $201 \mathrm{I}$ showing an $80-90 \%$ response rate (Ramsay and Leung, 2009). However, within 12-33 months, the disease almost invariably progresses to an androgen-independent or castrate-resistant state. After this, treatment is limited and death occurs within a few years (Ramsay and Leung, 2009). Alternative treatment is therefore required for advanced $\mathrm{PCa}$.

Advances in molecular biology have increased our understanding of the numerous molecular pathways that may contribute to prostate carcinogenesis. Careful analysis of these signalling pathways may facilitate the development of targeted therapies. Recently, our laboratory showed the importance of the MEK5/ ERK5 (mitogen/extracellular signal-regulated kinase 5/extracellular signal-regulated protein kinase 5) cascade in PCa (Mehta et al, 2003; McCracken et al, 2008). Extracellular signal-regulated protein kinase 5 belongs to the family of mitogen-activated protein kinases (MAPKs). The other main subfamilies are ERK1/2, c-Jun NH2-terminal protein kinases and p38 MAPKs (Chang and Karin, 2001). All are activated by extracellular stimuli such as growth factors and environmental stresses, and are recognised to control cell proliferation, migration and differentiation. Extracellular signal-regulated protein kinase 5 is the specific substrate of 
MEK5, and is regulated by mitogens (EGF and G-CSF), cytokines (LIF and CT-1) and stress $\left(\mathrm{H}_{2} \mathrm{O}_{2}\right.$ and sorbitol) (Ranganathan et al, 2006). Downstream effectors of the ERK5 cascade are not fully defined, but include myocyte enhancement factor (MEF) 2, Sap1 and c-Myc (Wang and Tournier, 2006). Our laboratory recently proposed ERK5 to be clinically significant in $\mathrm{PCa}$, its cytoplasmic expression in the primary tumour correlating with Gleason sum score $(P<0.0001)$. Interestingly, significant nuclear localisation of ERK5 was strongly associated with both unfavourable patient survival outcome and the development of castrate-resistant disease $(P<0.0001)$ (McCracken et al, 2008).

This study aimed to define the importance of ERK5 signalling in prostate carcinogenesis, by examining ERK5-mediated function by manipulation of its expression/function as well as evaluating nuclear ERK5 expression in primary and metastatic clinical PCa specimens.

\section{MATERIALS AND METHODS}

\section{Cell culture, siRNA transfection experiments and western blotting}

Cells are maintained and used as described previously: PC3, PC3derived ERK5 overexpressing (PC3-ERK5) cells and inducible HEK293 cells expressing MEK5D (a constitutively active mutant of MEK5) (Mehta et al, 2003; McCracken et al, 2008). Extracellular signal-regulated protein kinase 5 knockdown was carried out as reported by Sharma and Goalstone (2005). Extracellular signalregulated protein kinase 5 -specific siRNA ( $5^{\prime}$-GGTGTTGGCTTTG ACCTGGAGGAAT- $3^{\prime}$ ) was used (Eurogentec, Southampton, UK), and all experiments were performed at $10 \mathrm{~nm}$ siRNA concentration. Non-silencing (or scrambled) siRNA was included as controls.

Western blot analysis was performed as described previously (Mehta et al, 2003; McCracken et al, 2008). Antibodies were used at the following dilutions: anti-ERK5 1:500, anti-phospho ERK5 $1: 500$, p44/42 MAP kinase 1:1000, anti-phospho p44/42 MAP kinase (Cell Signalling Technology, Hertfordshire, UK) and antialpha tubulin 1:8000 (Santa Cruz Biotechnology, Santa Cruz, CA, USA). Horseradish peroxidase-conjugated secondary antibodies (Cell Signalling Technology) were applied at 1:3000 and detected using the enhanced chemiluminescence detection kit (Amersham, Piscataway, NJ, USA).

\section{Cell proliferation}

In all, $1.5 \times 10^{5}$ cells were seeded in six-well plates. siRNA transfection was performed and serial cell counts were taken at 24, 48, 72 and $96 \mathrm{~h}$ using a Casey cell counter. Each experiment was repeated at least three times, and at least three wells were used per condition each time. Where appropriate, MEK inhibitor was used in place of siRNA transfection.

\section{Cell tracking, chemotaxis migration and chemo-invasion assays}

Cell tracking motility assays were performed $48 \mathrm{~h}$ post-transfection with ERK5 siRNA. PC3-ERK5 or PC3 cells were studied using a Nikon (Melville, NY, USA) TE2000 time lapse microscope $(\times 20$ magnification). Images were taken every $15 \mathrm{~min}$ over an $18 \mathrm{~h}$ period. Image J software (Bethesda, MD, USA) was used to track random cellular motility; accumulated and euclidean (direct) distance were measured. At least eight cells were tracked per field and three fields were viewed in each experiment which was repeated twice.

In addition, the migrative potential of PC 3 cells, \pm ERK5 and \pm PD184352 was assessed using the chemotaxis-based BD-Falcon Transwell system (San Jose, CA, USA). Cells $\left(8 \times 10^{4}\right)$ were resuspended in $500 \mu \mathrm{l}$ basal medium \pm PD184352 and placed in the upper Transwell compartment. In all, $750 \mu \mathrm{l}$ of $\mathrm{BM} \pm \mathrm{EGF}$ $\left(100 \mathrm{ng} \mathrm{ml}^{-1}\right)$ was placed in the lower compartment to act as a potential attractant. After $24 \mathrm{~h}$ incubation at $37^{\circ} \mathrm{C}$ and $5 \% \mathrm{CO}_{2}$, cells that had not migrated were removed from the upper face of the membranes with cotton buds. The filters were fixed in methanol for $30 \mathrm{~min}$ at $-20^{\circ} \mathrm{C}$, stained with Haematoxylin Harris' (VWR International Ltd, Leicestershire, UK) for $1 \mathrm{~h}$ at room temperature and then washed carefully in $\mathrm{dH}_{2} \mathrm{O}$. After air dried, the membranes were mounted in DPX. The average number of cells per field of view (eight random fields per membrane) was counted under a light microscope at $\times 20$ magnification. For chemo-invasion assays, the same protocol was employed with Biocoat Matrigel invasion chambers in duplicate (Becton Dickinson Labware, San Jose, CA, USA; cat. no.: 354480). Each experiment was repeated at least three times and each time the mean numbers of migrated or invading cells were taken from three chambers. Similarly, invasion assay with siRNA-transfected PC3 or PC3-ERK5 cells were studied as above.

\section{MMP promoter studies}

At $48 \mathrm{~h}$ before transfection, $3 \times 10^{4}$ PC3 cells were seeded per well in a 48-well plate. Matrix metalloproteinase (MMP)9 (-670), MMP2 (-1659) and MMP1 (-512) constructs containing $5^{\prime}$-flanking fragments upstream to the transcription initiation start site linked to a luciferase reporter gene (MMP-luc) were co-transfected with ERK5 or empty plasmid (pcDNA3.1) using Superfect reagent, according to the manufacturer's recommendation. After $24 \mathrm{~h}$, cells were serum starved and left for another 16-24h before luciferase activities were determined. Cells were co-transfected with a $\beta$-gal-CMV plasmid to allow normalisation of transfection efficiency. A total of $300 \mathrm{ng}$ of DNA were transfected, containing $100 \mathrm{ng}$ MMP-luc, $150 \mathrm{ng}$ MEK5D and $50 \mathrm{ng} \beta$-galactosidase reporter. The $M M P 1 / M M P 2$ and $M M P 9$ promotor constructs were obtained as kind gifts from Dr Y Sun (Parke Davies Pharmaceutical Research, UK) and Dr D Boyd (MD Anderson Cancer Centre, USA), respectively.

\section{Quantitative real-time RT - PCR to profile MMP and TIMP expression}

Design of specific primers for human MMP and tissue inhibitors of metalloprotease (TIMP) genes and respective PCR reactions were performed as described (Altschul et al, 1990; Nuttall et al, 2003), with each PCR reaction containing $5 \mathrm{ng}$ of reverse transcribed RNA in $25 \mu \mathrm{l}$. The identity of PCR products was confirmed by direct sequencing of the amplicon. The $18 \mathrm{~S}$ ribosomal RNA gene was used as an endogenous control to normalise for differences in the amount of total RNA in each sample, using previously validated procedures (Wall and Edwards, 2002; Nuttall et al, 2003). To determine the relative RNA levels within the samples, standard curves for the PCR reaction were prepared by using the cDNA from one sample and making two-fold serial dilutions covering the range equivalent to $20-0.625 \mathrm{ng}$ of RNA (for $18 \mathrm{~S}$ analyses, the range was $4-0.125 \mathrm{ng}$ ).

\section{Invadopodia assay}

In vivo assessment of invadopodia was performed as described (Ayala et al, 2008). In brief, A375MM or PC3 cells were seeded onto gelatin-coated coverslips at a density of $1 \times 10^{4}$ or $2 \times 10^{4}$, respectively. Drug treatment groups were as follows: controls (no treatment or DMSO, $7.5 \mu \mathrm{lml}^{-1}$ ), $5 \mu \mathrm{M} \mathrm{PD18435}$ and $15 \mu \mathrm{M}$ PD18435. For ERK5 knockdown experiments, A375 cells were transfected with either ERK5-specific or scrambled siRNA before plating onto gelatin-coated coverlips. PC3 cells were transfected with ERK5 as described previously (McCracken et al, 2008). After $24 \mathrm{~h}$, cells were fixed for $20 \mathrm{~min}$ in $4 \%$ paraformaldehyde, washed three times in PBS and blocked for 10 min in $\mathrm{NH}_{4} \mathrm{Cl}$. Cells were 
permeablised in $0.1 \%$ Triton/PBS for $4 \mathrm{~min}$ and then stained with 594-Phalloidin $(1: 100)$ for $30 \mathrm{~min}$. Cells were given three final washes in PBS followed by two washes in $\mathrm{dH}_{2} \mathrm{O}$ and then mounted onto labelled glass slides.

A Zeiss (Thornwood, NY, USA) Axiopskop was used to capture images of at least 20 cells per test condition. For each cell, the red and green channels were used to capture the actin cytoskeleton and the gelatin layer under the cell showing any degradation, respectively. Image $\mathrm{J}$ and a plugin were used to evaluate these images and return a measure of degradation in terms of pixels. Two-tailed Student's $t$-test was performed on the data to establish levels of statistical significance in any differences observed.

\section{In vivo orthotopic prostate tumourigenesis study}

For orthotopic tumour growth, dorso-lateral prostates of male CD-1 nude mice were injected with either PC3-ERK5 cells (stably transfected with transfected pEGFP-C1-ERK5) or the control cells (stably transfected with the empty plasmid pEGFP-C1 vector) at $2.4 \times 10^{6}$ cells per $25 \mu \mathrm{l}$ serum free RPMI as previously described (Somers et al, 2003). Tumours were grown over a period of 12 weeks. The prostates were excised, weighted and fixed overnight in formalin before embedded in paraffin. All prostates were processed and cut in the same manner by a single histology technician to aid standardisation. All experiments were carried out in accordance with UK animal regulations.

\section{Immunohistochemical analysis}

Prostate cancer tissue microarray (TMA) was supplied by the University of Michigan Prostate Specialised Programme of Research Excellence. Tissues were obtained through either prostatectomy or rapid autopsy program with appropriate Internal Review Board approval as previously described (Rubin et al, 2000). Tissue microarray contained benign (normal) prostate samples $(n=30)$, benign prostatic hypertrophy $(\mathrm{BPH})$ samples $(n=27)$, prostatic intraepithelial neoplasia (PIN) $(n=24), 80$ cases of prostatic cancer as well as 32 metastatic lesions (lymph node $(n=10)$, liver samples $(n=17)$, lung $(n=2)$ and soft tissue $(n=3))$. Full tissue sections of benign and tumour human prostate were also used for optimisation of immunohistochemistry and in situ hybridisation. All tissue samples were radical prostatectomy samples, collected and used according to the ethical guidelines and procedures approved by the institutional supervisory committee. Immunohistochemistry for ERK5 expression was performed based on previously described protocol (Gnanapragasam et al, 2003). Immunoreactivity signals were assessed by independent observers (Maria Soofi and Morag Seywright) blinded to the clinical details. Nuclear ERK5 immunoreactivity was measured on histoscore scale from 0 (no staining) to 300 (very strong staining). In situ hybridisation for mir143 was performed as previously reported (Clape et al, 2009).

\section{Statistical analysis}

Data are shown as the mean \pm s.d. where possible and statistical analysis was obtained using SPSS version 15.0, computer software (SPSS Inc., Chicago, IL, USA). A $P$-value of $<0.05$ was taken to indicate statistical significance. Mann-Whitney test was used to test for differences of ERK5 immunoreactivity in different histological groups of prostate pathology. Chi-square test was used to assess data on metastasis in the orthotopic prostate model.

\section{RESULTS}

\section{Suppression of ERK5 function impaired the invasive capacity of PCa cells}

Successful knockdown of endogenous and exogenous ERK5 was achieved in PC3 and PC3 cells stably transfected with ERK5
(PC3-ERK5) respectively (Figure 1A). Expression of both endogenous and transfected ERK5 was significantly reduced, with evidence of suppressed pERK5 level following reduced ERK5 expression. Following transfection with siRNA targeting ERK5, suppression of ERK5 expression was sustained for at least up to $72 \mathrm{~h}$, with around $70 \%$ reduction in ERK5 expression. Extracellular signal-regulated protein kinase 5 targeting siRNA was shown to be specific: throughout the duration of the experiment, ERK1/2 expression was not affected by siRNA targeting ERK5.

As ERK5 overexpression has been shown to be associated with increased proliferation in vitro (McCracken et al, 2008;

\section{A}

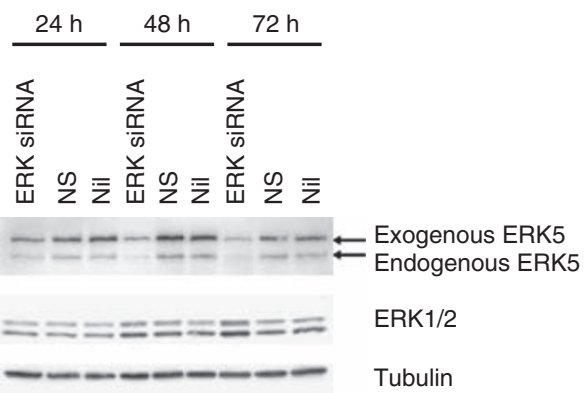

PC3 cells

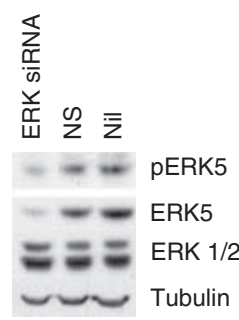

B
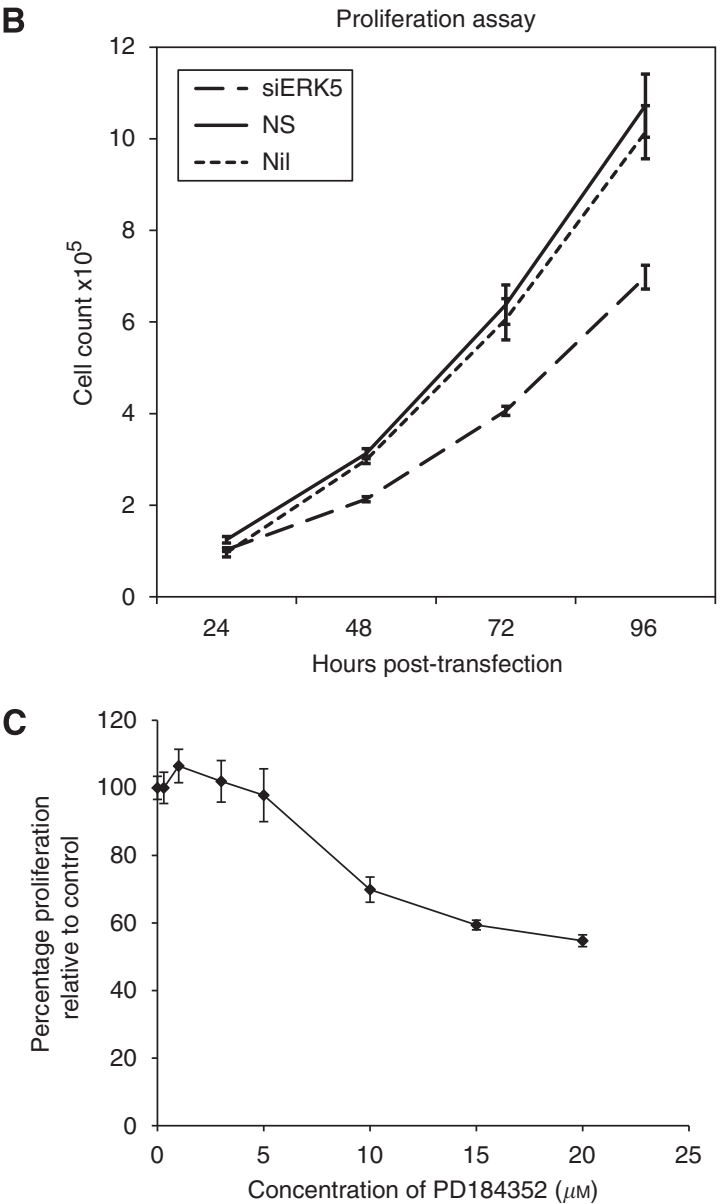

Figure I (A) Extracellular signal-regulated protein kinase 5 expression in PC3-ERK5 stable clone (left-hand panel) and parental PC3 cells (righthand panel) following siRNA-mediated ERK5 directed knockdown (KD), compared with non-silencing control (NS) and sham transfection (Nil). (B) Proliferation assay following ERK5 KD in PC3-ERK5 cells. (C) Effects of a range of doses of PD 84352 (MEK inhibitor) on proliferation of PC3 cells. 
Zen et al, 2009), we tested the effects of siRNA-mediated ERK5 knockdown on proliferation as well as motility and invasion. In PC3-ERK5 cells, a significant reduction in the rate of proliferation was observed on ERK5 knockdown, when compared with the controls (non-silencing siRNA and sham transfection controls respectively) at $96-\mathrm{h}$ post-transfection $(P<0.005$; Figure $1 \mathrm{~B})$.

To further assess the potential mitogenic effects of ERK5 signalling in prostate carcinogenesis, the effects of suppressed endogenous ERK5 expression by siRNA or function by the MEK inhibitor PD184352 were investigated in a proliferation assay using PC3 parental cells. Contrast to the data on PC3-ERK5 cells, no convincing anti-proliferative activities was observed neither with ERK5-targeted siRNA knockdown nor with MEK inhibitor PD184352 (Figure 1C and unpublished data). Even at relatively high dose of PD184352 (up to $20 \mu \mathrm{M}$ ), the reduction of proliferation has yet to reach $50 \%$; at these high doses, off-target effects are likely to contribute to the observed anti-proliferative effects. It should be noted that, in PC3 cells, PD184352 blocked phosphorylation of ERK1 at $0.3 \mu \mathrm{M}$ and ERK5 at $3 \mu \mathrm{M}$, respectively (Supplementary Figures $1 \mathrm{~A}$ and B), similar doserelated effects were observed in HEK293 cells (Supplementary Figure 1C).

We therefore hypothesised that ERK5 signalling may contribute to prostate carcinogenesis by driving cellular motility and/or invasion rather than proliferation. Random cellular motility was studied using real time cell tracking. Targeted knockdown of ERK5 expression by siRNA significantly decreased cell motility when compared with the non-silencing siRNA and untransfected cells. Untransfected and control transfected PC3-ERK5 cells display high levels of random motility. Both accumulated and euclidean distances following ERK5 knockdown were significantly reduced when compared with the controls $(P<0.005$; Figure $2 \mathrm{~A})$. Similarly, siRNA-mediated knockdown of ERK5 expression in PC3 cells reduced its motility (euclidean distance, Supplementary Figure 2). In keeping with our previous observation (McCracken et al, 2008), using an in vitro chemo-invasion matrigel assay, PC3-ERK5 cells have an enhanced invasive capability when compared with PC 3 cells, with $25(27 \pm 15)$ and $10(10 \pm 4)$ cells per field, respectively. On transfection with ERK5-targeted siRNA, the invasive capability of PC3 cells was significantly inhibited by 2.1- and 1.97-fold when compared with control siRNA-transfected and -untransfected PC3 cells, respectively $(P<0.005$; Figure $2 \mathrm{~B})$. As expected, PC3-ERK5 cells are more dependent on ERK5 signalling, and showed a more dramatic suppression of cellular invasion on ERK5 knockdown, when compared with the parental PC3 cells, with a 3.5- and 3.4-fold suppression in invasion $(P<0.005$; Figure 2B).

To confirm the data from ERK5 knockdown experiments, MEK1 inhibitor PD184352 was used to suppress ERK1/2 activation alone at $0.3 \mu \mathrm{M}$ and ERK-1, -2 and -5 activities at a higher concentration of $3 \mu \mathrm{M}$ before stimulation with EGF $\left(100 \mathrm{ng} \mathrm{ml}^{-1}\right.$ ) (Mody et al, 2001). Assays for chemotaxis and chemo-invasion were performed. In PC3 cells, 0.3 and $3 \mu \mathrm{M}$ PD184352 significantly suppressed ERK1/2 alone and ERK-1, -2 and -5 functions, respectively (Supplementary Figures 1A and B). Inhibition of ERK1/2 alone (PD184352 at $0.3 \mu \mathrm{M})$ did not affect EGF-mediated cellular migration and invasion in PC3 cells, but at pan ERK1/2/5 inhibiting dose of PD184352 $(0.3 \mu \mathrm{M})$, EGF-induced migration and invasion were significantly decreased $(P<0.001$, respectively; Figure 2C).

\section{ERK5-mediated invasive phenotype in vitro and in vivo}

MMP promoter luciferase constructs for MMP1, MMP2 and MMP9 were studied in the presence of transiently transfected ERK5 expression in PC3 cells. Matrix metalloproteinase-1, -2 and -9 were selected as they have been previously implicated in MEK5 signalling (Mehta et al, 2003). On ERK5 transfection, transcriptional activities of the $M M P-1,-2$ and -9 promoters (corrected for $\beta$-galactosidase and empty vector contsrol) were induced by 3.89-, 5.61- and 4.04-fold, respectively $(P<0.001$ for all three experiments, Figure $3 \mathrm{~A})$. In addition, a PCR-based expression analysis for a panel of proteases was then performed using HEK293 cells stably expressing an inducible constitutively active MEK5 mutant (MEK5D-HA) with negligible background MEK5 expression (Mehta et al, 2003). Triplicate samples of uninduced and induced cells were analysed. Consistent with the promoter luciferase data, on activation of MEK5/ERK5 signalling, MMP2 and MMP9 mRNA expression were upregulated by 1.8 - and 2.1-fold, respectively, although no increase was seen in MMP1 mRNA expression (Supplementary Table). In addition, MMP12 and TIMP2 expression were upregulated by 2.7 - and 2.2-fold, respectively, with MMP16 expression reduced by 1.7 -fold. We therefore confirmed MMP2 and MMP9 as target MMPs in MEK5/ ERK5 signalling, and identified MMP12, MMP16 and TIMP2 as novel potential proteases and regulator downstream of the MEK5/ ERK5 pathway. Their role in the ERK5-driven invasive phenotype warrants further investigation.

Invadopodia are proteolytically active protrusions formed by invasive tumour cells when grown on an extracellular matrix (ECM) substratum ((Ayala et al, 2008) and reference within). On the basis of our data on ERK5-mediated invasion and MMP expression, we hypothesised that suppression of ERK5 function by PD18435 at an ERK5 inhibitory dose will significantly suppress invadopodia formation. This is particularly relevant as ERK1/2 has been implicated to have a key regulatory input in invadopodia formation and ECM degradation (Ayala et al, 2008). The human malignant melanoma A375 cancer cells are well established for invadopodia assay (see Supplementary Figure 3) and were plated on gelatin and cultured for $24 \mathrm{~h}$ in the presence of either PD18435 (at 5 or $15 \mu \mathrm{M}$ ) or the corresponding concentration of DMSO (control). A375 cells treated with $15 \mu \mathrm{M}$ PD18435 (an inhibitory dose for pan ERK1/2/5) showed significantly less gelatin degradation when compared with the vehicle control $(P=0.0014)$ and ERK1/2 suppressing dose of PD18435 ( $5 \mu \mathrm{M} ; P<0.001)$ (Figure 3B). Furthermore, siRNA-mediated knockdown of ERK5 in A375 cells also significantly suppressed their ability to form invadopodia (Figure 3D). PC3 cells form invadopodia much less efficiently than A375 cells, generally producing a low level of diffuse signal (Supplementary Figure 3). Hence, ERK5 was transfected into PC3 cells to test if ERK5 overexpression will promote invadopodia formation. Transfected ERK5 expression in PC3 cells significantly enhanced invadopodia formation $(P<0.005$, Figure $3 C)$.

The in vivo impact of ERK5 on the invasiveness of prostate tumourigenesis was further investigated in an orthotopic prostate tumour model. PC3-ERK5 cells or the empty vector control cells were injected into the dorso-lateral prostate of nude mice $(n=8$ per group). As expected, after 12 weeks, tumour incidence in both cohorts was $100 \%$ (16 out of 16 ) with a statistically insignificant difference in their respective increase in tumour burden (as assessed by prostate weight) (Figure 3E). In keeping with ERK5's role in promoting an invasive phenotype, a statistically significant increase in metastases to lymph nodes (4 out of $8 v s 0$ out of 8 ) and lung ( 3 out of $8 v s$ out of 8 ) was observed in the PC 3 - ERK5 cells when compared with control cells.

\section{ERK5 expression in clinical PCa}

Recent studies have implicated ERK5 signalling in invasive PCa. However, ERK5 expression has not been formally tested in metastatic PCa. Our in vitro data would suggest that upregulated ERK5 signalling may contribute to an aggressive cancer phenotype including metastatic disease. Therefore, 32 cases of metastatic prostate tumours were examined for ERK5 immunoreactivity, along with normal prostate, $\mathrm{BPH}$, precursor lesion (PIN) and primary $\mathrm{PCa}$. Both $\mathrm{BPH}$ and normal prostate samples expressed 

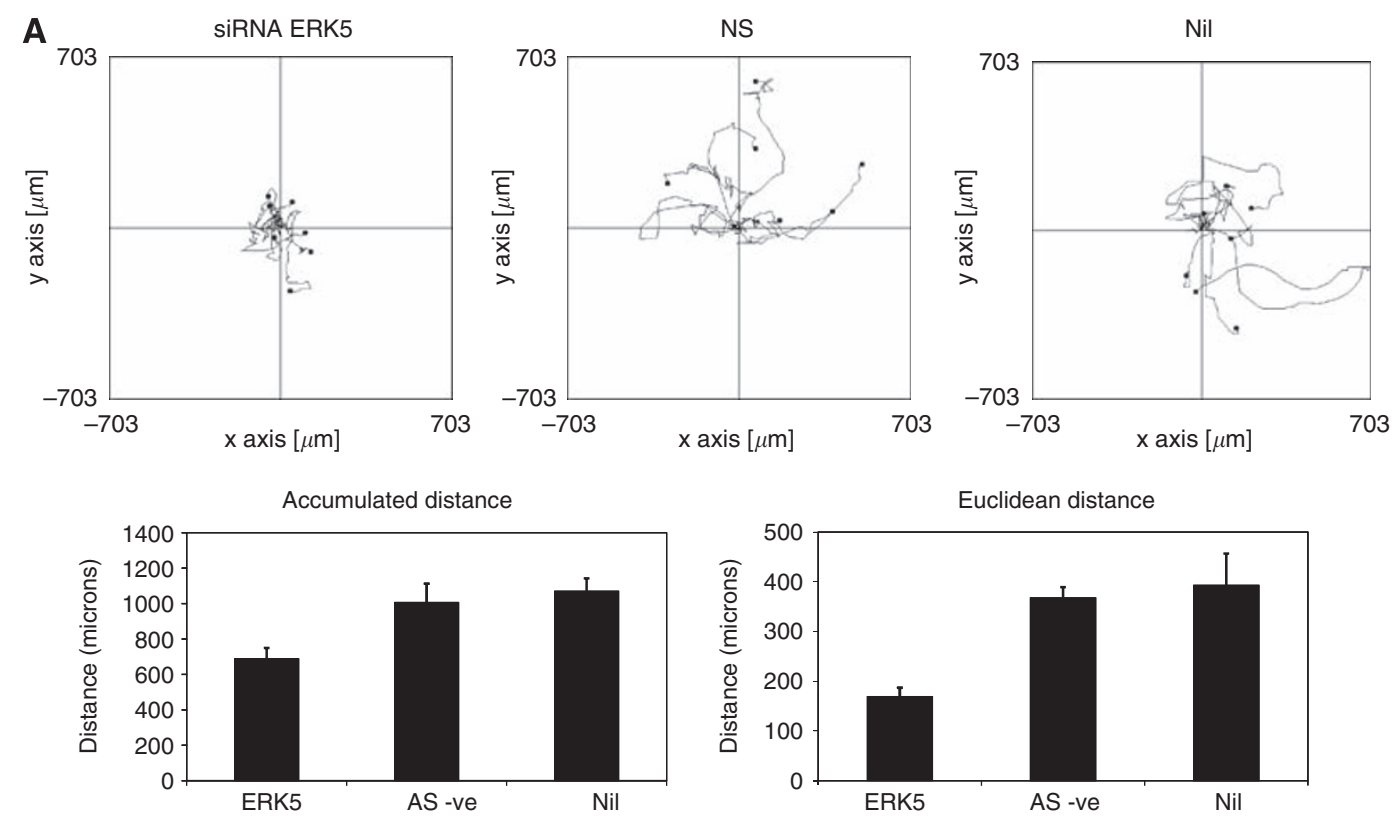

B
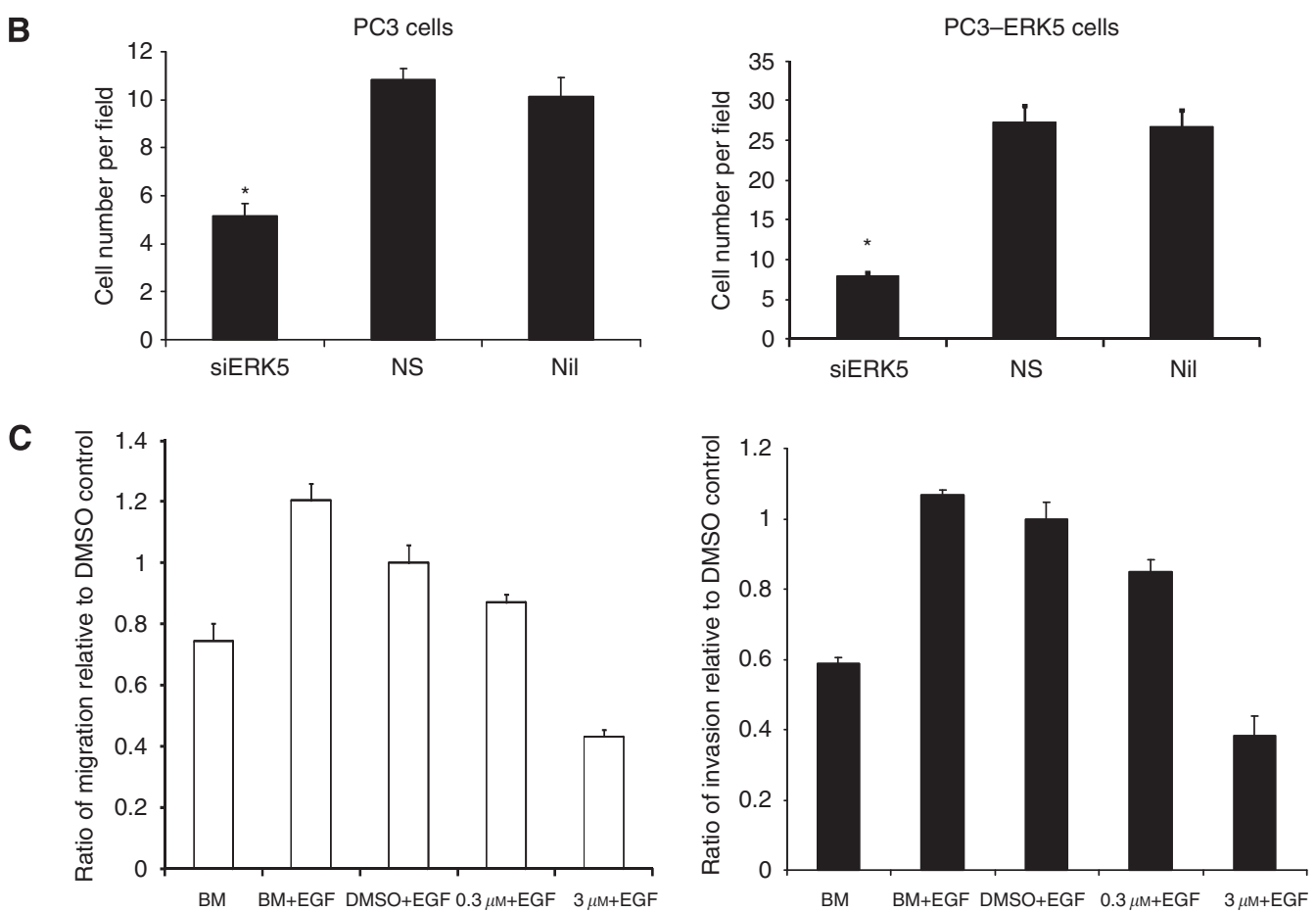

Figure 2 (A) Suppression PC3-ERK5 cellular motility by siRNA-mediated ERK5 KD (siERK5) when compared with control siRNA (NS, non-silencing) transfection or sham transfection $(\mathrm{Nil})\left({ }^{*} \mathrm{P}<0.005\right)$. Top panel showing the 'spider plot' of motile pattern of tracked cells; bottom panel shows overall distance travelled (accumulated and euclidean distances). (B) Suppression of cellular invasion in PC3 and PC3-ERK5 cells following siRNA-mediated ERK5 KD. (C) PDI 84352 at ERK5 suppressing dose of $3 \mu \mathrm{M}$ inhibited EGF-driven cellular migration and invasion in PC3 cells.

ERK5 at similar levels. Consistent with previous data, there was a trend for high levels of cytoplasmic ERK5 expression in tumours with high Gleason sum score $(>7)$ and high serum PSA levels $(>10)$ (data not shown). Importantly, nuclear ERK5 immunoreactivity in metastatic $\mathrm{PCa}$ was significantly stronger than that observed in $\mathrm{BPH}$ and primary $\mathrm{PCa}(P=0.013$ and $P<0.0001$, respectively, Mann-Whitney test; Figures $4 \mathrm{~A}-\mathrm{D}, \mathrm{G}$ and $\mathrm{H}$ ). Pre-malignant PIN lesions also showed significant upregulation of ERK5 expression, when compared with BPH control $(P=0.015$, Mann - Whitney test; Figure 4E). This would suggest involvement of ERK5 to be an early event in prostate carcinogenesis.
The mechanism for ERK5 activation in PCa remains to be fully examined. MicroRNA mir143 expression has recently been implicated to regulate ERK5 expression (Clape et al, 2009). Qualitative analysis of mir143 mRNA and ERK5 protein expression was performed on sequential sections from selected cases of PCa $(n=3)$. In keeping with published data, an inverse relationship between mir143 and ERK5 expression was noted, namely reduced mir143 and enhanced ERK5 expression in the malignant epithelium (and vice versa for benign tissue; Figures $4 \mathrm{I}$ and J). In addition, mir143 expression was seen in benign prostatic glands, endothelium (Figure $4 \mathrm{~K}$ ) and stromal smooth muscle cells. 
A

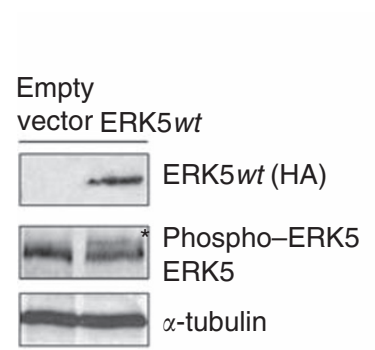

C

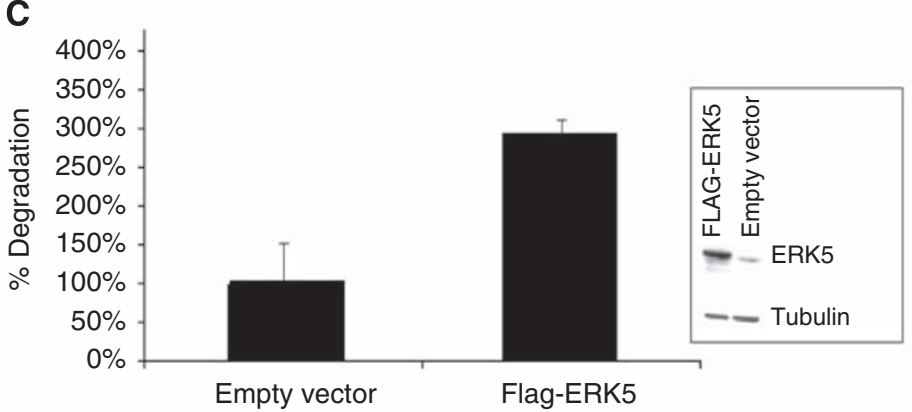

D

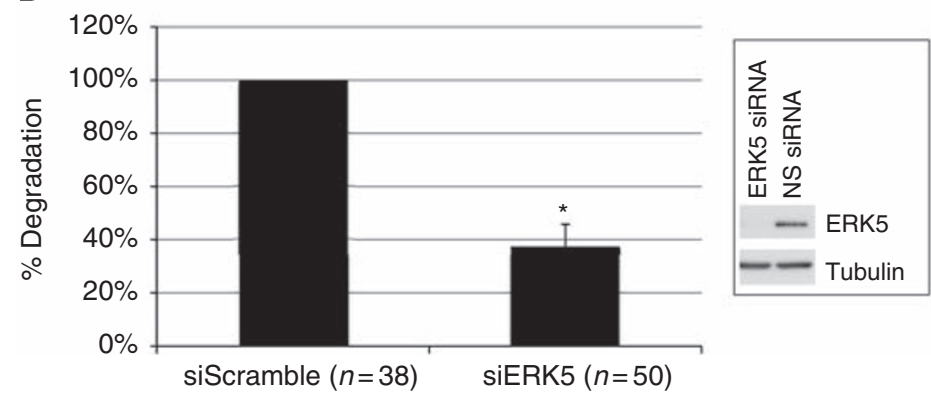

B

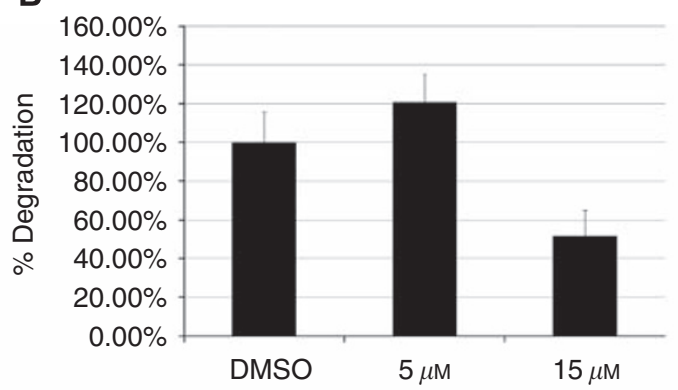

E

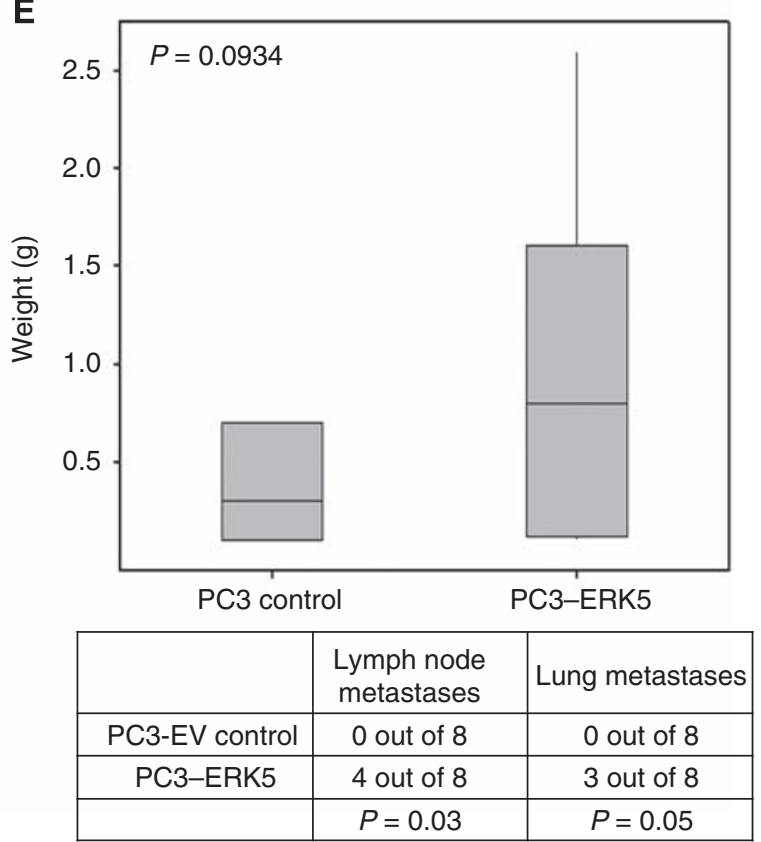

Figure 3 (A) Extracellular signal-regulated protein kinase 5-induced MMP promoter activity - (left panel) ERK5 DNA was transiently transfected into PC3 cells where a band (*) corresponding to phospo-ERK5 was seen on stimulation with EGF. (right panel) PC3 cells were co-transfected with MMPI, MMP2 or MMP9 promoter luciferase constructs along with ERK5 or empty plasmid. Transfection efficiency was assessed and normalised to $\beta$-galactosidase activity. Data represent mean fold induction compared with basal level of promoter activity from sets of quadruplicate assays \pm s.e. Experiments were performed three times. (B) Invadopodia formation by A375MM cells is significant suppressed by treatment with I5 $\mu$ M (but not $5 \mu \mathrm{M})$ PDI84352 ( $P<0.005$ ). (C) Transfected ERK5 significantly drives invadopodia formation in PC3 cells $(P<0.005)$. Insert of western blot shows upregulated ERK5 expression in PC3 cells. (D) Invadopodia formation in A375MM cells is significantly suppressed by siRNA-mediated knockdown of ERK5 expression (*P <0.05). Control cells were transfected with non-silencing scrambled siRNA. Number in brackets denotes the cells studied for each condition. Insert of western blot shows reduced ERK5 expression following siRNA transfection. (E) PC3-ERK5 cells form significantly more metastatic lesions (lymph nodes and lung) than control cells in an orthotopic prostate model $(P=0.03$ and $P=0.05$ respectively).

Taking together, our data on ERK5 function in vitro and in vivo as well as expression in clinical PCa support its role in an invasive phenotype.

\section{DISCUSSION}

In this report, we showed the first evidence for upregulated ERK5 expression in metastatic PCa. Upregulated ERK5 expression in PIN lesions would suggest an early involvement of ERK5-mediated signalling in prostate carcinogenesis. Amplification or mutation of the MEK5 and ERK5 genes is infrequent; within the data available at catalogue of somatic mutations in cancer (COSMIC, Sanger Institute, UK; www.sanger.ac.uk/genetics/CGP/cosmic), they are relatively uncommon events seen in skin, liver and breast cancer. Our finding of reduced mir143 expression with corresponding enhanced ERK5 expression in sequential tissue sections strongly argue for a role of mir143 at least partly contributing to abnormal
ERK5 expression in PCa. Future studies are warranted to validate and define the mechanism for loss of mir143 expression.

Recent data have been accumulating to support a key role of MEK5/ERK5 signalling in carcinogenesis. Although MEK5/ERK5 signalling clearly promotes cell cycle promotion in certain context (Kato et al, 1997, 1998), there are situations where ERK5 function does not contribute to proliferation (Squires et al, 2002; Wang et al, 2005). Abnormal signalling by MEK5/ERK5 has been implicated in a number of tumour types. Amplification of the ERK5 gene locus has been reported in hepatocellular carcinoma where ERK5 function appears to be a key mitogenic factor (Zen et al, 2009). In contrast, our data in PCa did not suggest significant mitotic advantage as a result of ERK5 function. Instead, ERK5-mediated signalling appears to critically regulate cellular motility and invasion in $\mathrm{PCa}$, which is in keeping with our observed association between aberrant ERK5 expression in the primary prostate tumours and a less favourable survival outcome (McCracken et al, 2008). 

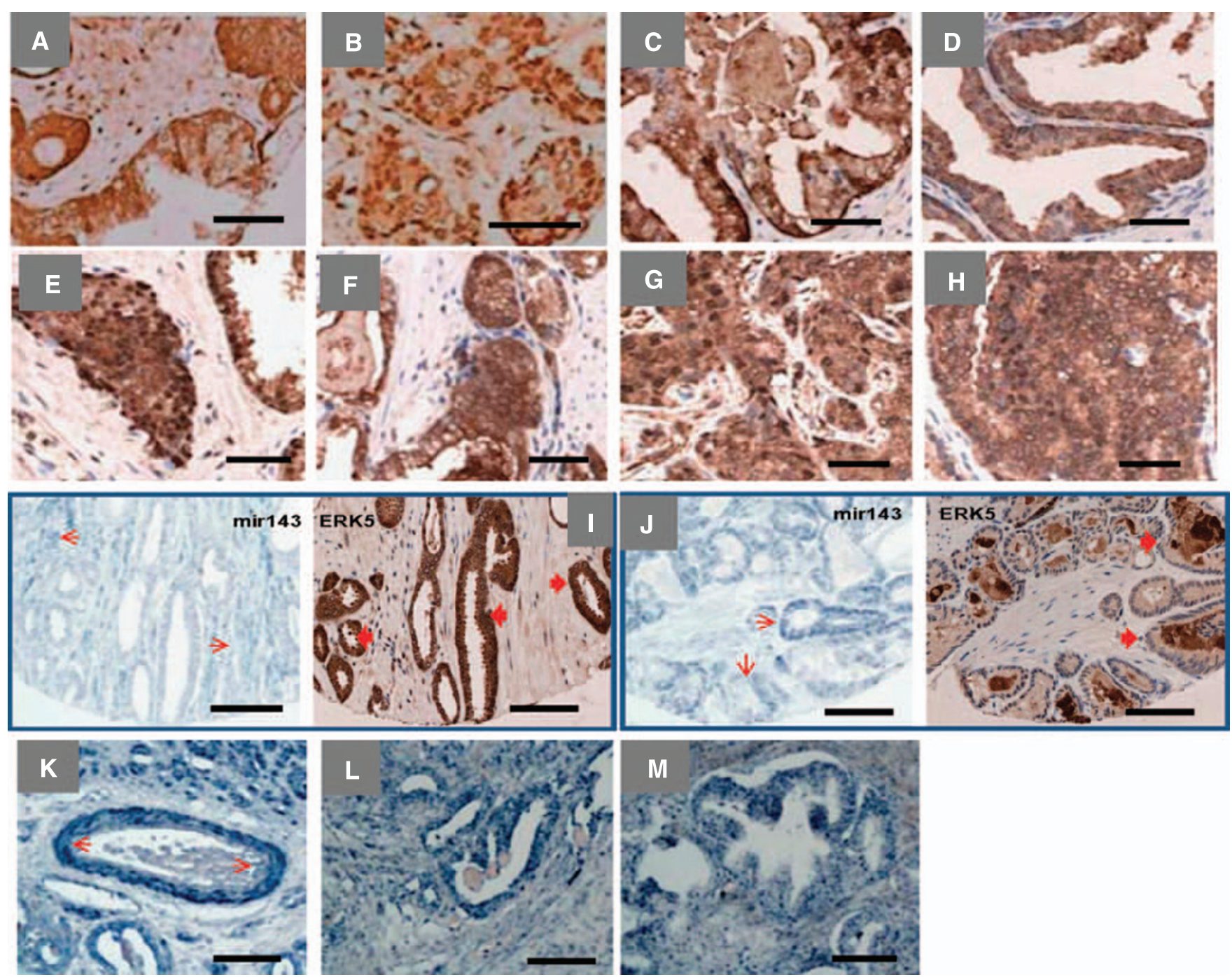

Figure 4 Extracellular signal-regulated protein kinase 5 protein and mirl43 transcript expression in clinical prostate cancer (PCa) by immunohistochemistry $(\mathrm{IHC})$ and in situ hybridisation $(\mathrm{ISH})$ respectively. Extracellular signal-regulated protein kinase 5 immunoreactivity in $(\mathbf{A})$ Gleason 7 PCa, (B) Gleason 9 PCa, (C) BPH, (D) normal prostate, (E) PIN, (F) Castrate-resistant PCa (CRPC), (G) liver metastasis, (H) lymph node metastasis; (I and J) mirl 43 mRNA and ERK5 protein expression analysis in sequential slides from two cases of PCa (solid and open arrows signify ERK5 protein and mirl43 transcript expression, respectively), (K) mirl43 expression in endothelium; $\beta$-actin expression in (L) BPH and (M) PCa (scale bar represents $100 \mu \mathrm{m})$.

Degradation of the ECM is integral to tumourigenesis and is mediated by the activity of ECM proteases, including the MMPs and the serine proteases (Sternlicht and Werb, 2001). There are at least 25 members of the MMP family; their functions include a role in ECM degradation in the context of tumour establishment: growth and migration, avoidance of apoptosis, angiogenesis and interaction with the immune system (Overall and Lopez-Otin, 2002). These effects are achieved in part by the cleavage of growth factors, their receptors, or other growth factor-associated proteins (Egeblad and Werb, 2002). An important means of their inhibition is achieved by the binding of the TIMPs, of which there are four members (Jiang et al, 2002).

There is considerable evidence supporting the involvement of MMPs and TIMPs in PCa (Pajouh et al, 1991; Trudel et al, 2003; Udayakumar et al, 2003). Of the known MMPs studied in the prostate, MMP9 overexpression correlates with metastatic disease. Levels of MMP9 mRNA and mature protein have both been shown to be elevated in malignant prostatic tissue, particularly in aggressive and metastatic tumours (Hamdy et al, 1994; Wood et al, 1997; Dong et al, 2001). For the first time, our data implicated expression of MMP12, 16 and TIMP2 to be regulated by ERK5-mediated signalling (Clark et al, 2008).

Taking our previous data on MEK5 (Mehta et al, 2003) and the current report on ERK5, activation of expression for members of the MMP family, particularly MMP9, by MEK5/ERK5 appear to be an important signalling events. The elements controlling the expression of $M M P 9$ have been well characterised and include AP-1, NF- $\kappa$ B, Ets and Sp1 sites, mainly within -670 -bp region upstream to the transcription start site (Sato and Seiki, 1993). In particular, both the AP-1 and NF- $\kappa \mathrm{B}$-binding sites have been shown to be important regulatory elements of the MMP9 promoter (Ricca et al, 2000; Troussard et al, 2000). Serum-induced activation of ERK5 results in MEF2C dependent transcriptional activation of c-jun to increase AP-1 levels (Kato et al, 1997).

In this report, we showed that upregulated ERK5 expression promoted MMP expression: (1) enhanced MMP-1, -2 and -9 promoter luciferase reporter activities in PC3 cells $(P<0.001$ for three constructs); (2) MMP and TIMP expression profiling by Q-RT - PCR in HEK293 cells (Supplementary Table 1) (Riddick et al, 2005) confirmed increased MMP2 and MMP9 expression 
(1.8- and 2.1-fold, respectively), although MMP1 mRNA expression was not affected. This apparent difference in MMP1 expression between the promoter reporter assay and the quantitative real time data may be due to the different cell lines used in the two experiments, namely PC3 and 293 cells. Evidence of in vivo ECM degradation at the site of invadopodia is interesting. Degradation of gelatin at these sites is consistent with ERK5-mediated upregulated expression of MMP-2 and -9, also referred to as the gelatinase group of MMPs. As MMP12 was significantly upregulated by ERK5 signalling, it is worth noting that MMP12 (or human macrophage elastase) is able to activate MMP2 to exaggerate the cascade of proteolytic processes (Chen, 2004). As our data have suggested effects at the transcription level, future studies to examine the impact of ERK5 signalling on protein expression and functional status of the key MMPs such as MMP9 are required.

Taken together, our in vitro, in vivo and clinical data support an important role for the MEK5-ERK5 signalling pathway in invasive $\mathrm{PCa}$ and may represent a potential target for therapy, including primary and metastatic PCa. In addition, the recent discovery of

\section{REFERENCES}

Altekruse SF, Kosary CL, Krapcho M (2010) SEER Cancer Statistics Review, 1975-2007. National Cancer Institute: Bethesda, MD http://seer.cancer. gov/csr/1975_2007/ (based on November 2009 SEER data submission, posted to the SEER web site, 2010)

Altschul SF, Gish W, Miller W, Myers EW, Lipman DJ (1990) Basic local alignment search tool. J Mol Biol 215: 403-410

Ayala I, Baldassarre M, Giacchetti G, Caldieri G, Tete S, Luini A, Buccione R (2008) Multiple regulatory inputs converge on cortactin to control invadopodia biogenesis and extracellular matrix degradation. J Cell Sci 121: $369-378$

Chang L, Karin M (2001) Mammalian MAP kinase signalling cascades. Nature 410: $37-40$

Chen YE (2004) MMP-12, an old enzyme plays a new role in the pathogenesis of rheumatoid arthritis? Am J Pathol 165: 1069-1070

Clape C, Fritz V, Henriquet C, Apparailly F, Fernandez PL, Iborra F, Avances C, Villalba M, Culine S, Fajas L (2009) miR-143 interferes with ERK5 signaling, and abrogates prostate cancer progression in mice. PLoS One 4: e7542

Clark IM, Swingler TE, Sampieri CL, Edwards DR (2008) The regulation of matrix metalloproteinases and their inhibitors. Int J Biochem Cell Biol 40: $1362-1378$

Dong Z, Nemeth JA, Cher ML, Palmer KC, Bright RC, Fridman R (2001) Differential regulation of matrix metalloproteinase-9, tissue inhibitor of metalloproteinase-1 (TIMP-1) and TIMP-2 expression in co-cultures of prostate cancer and stromal cells. Int J Cancer 93: 507-515

Egeblad M, Werb Z (2002) New functions for the matrix metalloproteinases in cancer progression. Nat Rev Cancer 2: 161-174

Gnanapragasam VJ, Robinson MC, Marsh C, Robson CN, Hamdy FC, Leung HY (2003) FGF8 isoform b expression in human prostate cancer. Br J Cancer 88: 1432 - 1438

Hamdy FC, Fadlon EJ, Cottam D, Lawry J, Thurrell W, Silcocks PB, Anderson JB, Williams JL, Rees RC (1994) Matrix metalloproteinase 9 expression in primary human prostatic adenocarcinoma and benign prostatic hyperplasia. Br J Cancer 69: 177 - 182

Jiang Y, Goldberg ID, Shi YE (2002) Complex roles of tissue inhibitors of metalloproteinases in cancer. Oncogene 21: 2245-2252

Kato Y, Kravchenko VV, Tapping RI, Han J, Ulevitch RJ, Lee JD (1997) BMK1/ERK5 regulates serum-induced early gene expression through transcription factor MEF2C. EMBO J 16: 7054-7066

Kato Y, Tapping RI, Huang S, Watson MH, Ulevitch RJ, Lee JD (1998) Bmk1/Erk5 is required for cell proliferation induced by epidermal growth factor. Nature 395: 713-716

McCracken SR, Ramsay A, Heer R, Mathers ME, Jenkins BL, Edwards J, Robson CN, Marquez R, Cohen P, Leung HY (2008) Aberrant expression of extracellular signal-regulated kinase 5 in human prostate cancer. Oncogene 27: 2978-2988

Mehta PB, Jenkins BL, McCarthy L, Thilak L, Robson CN, Neal DE, Leung HY (2003) MEK5 overexpression is associated with metastatic specific ERK5 inhibitor will provide the molecular probe to further evaluate the molecular basis of ERK5-driven carcinogenesis (Yang et al, 2010).

\section{ACKNOWLEDGEMENTS}

This work was supported by Cancer Research UK and Prostate Cancer Charity. AKR received a clinical research fellowship from the Royal College of Physicians and Surgeons of Glasgow. Image acquisition system following immunohistochemistry experiment was part funded by a grant from Think Pink Charity, UK. Tissue microarray was produced as part of the Specialised Programme of Research Excellence for Prostate Cancer (SPORE) National Cancer Institute (NCI) grant P50CA69568.

Supplementary Information accompanies the paper on British Journal of Cancer website (http://www.nature.com/bjc) prostate cancer, and stimulates proliferation, MMP-9 expression and invasion. Oncogene 22: $1381-1389$

Mody N, Leitch J, Armstrong C, Dixon J, Cohen P (2001) Effects of MAP kinase cascade inhibitors on the MKK5/ERK5 pathway. FEBS Lett 502: $21-24$

Nuttall RK, Pennington CJ, Taplin J, Wheal A, Yong VW, Forsyth PA, Edwards DR (2003) Elevated membrane-type matrix metalloproteinases in gliomas revealed by profiling proteases and inhibitors in human cancer cells. Mol Cancer Res 1: 333-345

Overall CM, Lopez-Otin C (2002) Strategies for MMP inhibition in cancer: innovations for the post-trial era. Nat Rev Cancer 2: 657-672

Pajouh MS, Nagle RB, Breathnach R, Finch JS, Brawer MK, Bowden GT (1991) Expression of metalloproteinase genes in human prostate cancer. J Cancer Res Clin Oncol 117: 144-150

Ramsay AK, Leung HY (2009) Signalling pathways in prostate carcinogenesis: potentials for molecular-targeted therapy. Clin Sci (London) 117: $209-228$

Ranganathan A, Pearson GW, Chrestensen CA, Sturgill TW, Cobb MH (2006) The MAP kinase ERK5 binds to and phosphorylates p90 RSK. Arch Biochem Biophys 449: 8-16

Ricca A, Biroccio A, Del Bufalo D, Mackay AR, Santoni A, Cippitelli M (2000) bcl-2 over-expression enhances NF-kappaB activity and induces mmp-9 transcription in human MCF7(ADR) breast-cancer cells. Int J Cancer 86: 188-196

Riddick AC, Shukla CJ, Pennington CJ, Bass R, Nuttall RK, Hogan A, Sethia KK, Ellis V, Collins AT, Maitland NJ, Ball RY, Edwards DR (2005) Identification of degradome components associated with prostate cancer progression by expression analysis of human prostatic tissues. Br J Cancer 92: $2171-2180$

Rubin MA, Putzi M, Mucci N, Smith DC, Woino K, Korenchuk S, Pienta KJ (2000) Rapid ('warm') autopsy study for procurement of metastatic prostate cancer. Clin Cancer Res 6: 1038-1045

Sato H, Seiki M (1993) Regulatory mechanism of $92 \mathrm{kDa}$ type IV collagenase gene expression which is associated with invasiveness of tumor cells. Oncogene 8: 395-405

Sharma G, Goalstone ML (2005) Dominant negative FTase (DNFTalpha) inhibits ERK5, MEF2C and CREB activation in adipogenesis. Mol Cell Endocrinol 245: $93-104$

Somers KD, Brown RR, Holterman DA, Yousefieh N, Glass WF, Wright Jr GL, Schellhammer PF, Qian J, Ciavarra RP (2003) Orthotopic treatment model of prostate cancer and metastasis in the immunocompetent mouse: efficacy of flt3 ligand immunotherapy. Int J Cancer 107: 773-780

Squires MS, Nixon PM, Cook SJ (2002) Cell-cycle arrest by PD184352 requires inhibition of extracellular signal-regulated kinases (ERK) $1 / 2$ but not ERK5/BMK1. Biochem J 366: 673-680

Sternlicht MD, Werb Z (2001) How matrix metalloproteinases regulate cell behavior. Annu Rev Cell Dev Biol 17: 463-516 
Troussard AA, Costello P, Yoganathan TN, Kumagai S, Roskelley CD, Dedhar S (2000) The integrin linked kinase (ILK) induces an invasive phenotype via AP-1 transcription factor-dependent upregulation of matrix metalloproteinase 9 (MMP-9). Oncogene 19: $5444-5452$

Trudel D, Fradet Y, Meyer F, Harel F, Tetu B (2003) Significance of MMP-2 expression in prostate cancer: an immunohistochemical study. Cancer Res 63: $8511-8515$

Udayakumar TS, Chen ML, Bair EL, Von Bredow DC, Cress AE, Nagle RB, Bowden GT (2003) Membrane type-1-matrix metalloproteinase expressed by prostate carcinoma cells cleaves human laminin-5 beta3 chain and induces cell migration. Cancer Res 63: 2292-2299

Wall SJ, Edwards DR (2002) Quantitative reverse transcription-polymerase chain reaction (RT-PCR): a comparison of primer-dropping, competitive, and real-time RT-PCRs. Anal Biochem 300: 269-273

Wang X, Merritt AJ, Seyfried J, Guo C, Papadakis ES, Finegan KG, Kayahara M, Dixon J, Boot-Handford RP, Cartwright EJ, Mayer U, Tournier C (2005) Targeted deletion of mek 5 causes early embryonic death and defects in the extracellular signal-regulated kinase 5/myocyte enhancer factor 2 cell survival pathway. Mol Cell Biol 25: 336-345

Wang X, Tournier C (2006) Regulation of cellular functions by the ERK5 signalling pathway. Cell Signal 18: $753-760$

Wood M, Fudge K, Mohler JL, Frost AR, Garcia F, Wang M, Stearns ME (1997) In situ hybridization studies of metalloproteinases 2 and 9 and TIMP-1 and TIMP-2 expression in human prostate cancer. Clin Exp Metastasis 15: 246-258

Yang Q, Deng X, Lu B, Cameron M, Fearns C, Patricelli MP, Yates III JR, Gray NS, Lee JD (2010) Pharmacological inhibition of BMK1 suppresses tumor growth through Promyelocytic Leukemia Protein. Cancer Cell 18: 258-267

Zen K, Yasui K, Nakajima T, Zen Y, Zen K, Gen Y, Mitsuyoshi H, Minami M, Mitsufuji S, Tanaka S, Itoh Y, Nakanuma Y, Taniwaki M, Arii S, Okanoue T, Yoshikawa T (2009) ERK5 is a target for gene amplification at 17p11 and promotes cell growth in hepatocellular carcinoma by regulating mitotic entry. Genes Chromosomes Cancer 48: 109-120 